\title{
RELIABILITY OF THE FAGERSTROM TOLERANCE QUESTIONNAIRE AND THE FAGERSTROM TEST FOR NICOTINE DEPENDENCE
}

\author{
CYNTHIA S. POMERLEAU, SOLANGE M. CARTON, MARY L. LUTZKE, \\ KAREN A. FLESSLAND, and OVIDE F. POMERLEAU \\ Behavioral Medicine Program, Department of Psychiatry, University of Michigan School of Medicine
}

\begin{abstract}
The purpose of this study was to assess the test-retest stability of the Fagerstrom Tolerance Questionnaire (FTQ) in two samples: (a) paid subjects in an American laboratory; data were collected via telephone screen and subsequently via questions embedded in a written history; and (b) smokers hospitalized for depression in Paris; data were collected via a written questionnaire upon admission and again after 3 weeks of treatment for depression. Reliability data are also presented for a recently revised version of the FTQ, the Fagerstrom Test for Nicotine Dependence (FTND), and compared with FTQ data collected in a subsample of subjects in the American database who received both versions of the questionnaire. Both the FTQ (in both samples) and the FTND proved to be highly reliable. The validity of the scales, using cotinine, number of years smoked, and the "addictive" factor on the Classification of Smoking by Motives questionnaire as criterion variables, was also supported. No relationship between FTQ score and severity of depression was detected in either sample. Internal consistency was somewhat higher for the FTND than for the FTQ, replicating previous findings in the literature.
\end{abstract}

What has come to be known as the Fagerstrom Tolerance Questionnaire (FTQ; Fagerstrom, 1978) was presented by the author as a test of "physical dependence to nicotine." Although he reported validation of his instrument in small samples using as his touchstones change in body temperature following withdrawal in smokers and heart rate increment during smoking in both smokers and exsmokers, it is probably safe to say that the widespread use that followed, in both research and clinical settings, was due less to its superior psychometric properties than to the fact that it filled a "market niche," at a time when the importance of nicotine dependence as an obstacle to quitting was becoming increasingly apparent and when pharmacotherapeutic approaches were starting to be developed. Its only "competitors" were questionnaires such as the Reasons for Smoking Scale (Ikard \& Tomkins, 1973; Tomkins, 1966) and the Classification of Smoking by Motives (CSM; Russell, Peto, \& Patel, 1974), which were intended to categorize smokers - or more accurately, smoking behavior - on the basis of smoking motivation typology. Though some of the types discriminated (e.g., the CSM "addictive" smoking; Russell et al., 1974) might be thought of as tapping a similar construct, these scales, unlike the FTQ, were not explicitly intended to provide a continuum along which all smokers could be placed.

Testing of the psychometric properties of the instrument has thus proceeded haphazardly, rather than systematically in different subject samples. Conspicuously lacking, for example, has been any attempt to establish the reliability of the scale.

Preparation of this manuscript was supported by Grant DA 06529 to the last author from the National Institute on Drug $A$ buse.

Requests for reprints should be sent to Cynthia S. Pomerleau, Behavioral Medicine Program, University of Michigan Department of Psychiatry, 475 Market Place, Suite L, Ann Arbor, MI 48108. 
Nevertheless, the FTQ has by and large acquitted itself fairly well (Fagerstrom \& Schneider, 1989), having been demonstrated to predict such measures as cotinine (Pomerleau, Pomerleau, Majchrzak, Kloska, \& Malakuti, 1990), CO (Fagerstrom, 1982), nicotine boost upon smoking (Lombardo, Hughes, \& Fross, 1988), and success in smoking cessation (Pinto, Abrams, Monti. \& Jacobus, 1987), and to distinguish between light and heavy smokers stratified on the basis of cotinine (Pomerleau. Fertig, \& Shanahan, 1983). Over the years, however, a number of shortcomings have been identified, especially relating to internal consistency (Lichtenstein \& Mermelstein, 1986; Swan, Ward, \& Jack, 1991) and to failure of some of the items to contribute to the predictive power of the test (Heatherton. Kozlowski. Frecker, \& Fagerstrom, 1991). These objections have led to suggestions for refinement of individual items (Moore, Schneider, \& Ryan, 1987; Pomerleau, Majchrzak, \& Pomerleau, 1989) and more recently to the proposal by Fagerstrom and a group of collaborators of a new version, the Fagerstrom Test for Nicotine Dependence (FTND; Heatherton et al., 1991).

The purpose of the present study was to fill a gap in the literature by determining the reliability of the FTQ in two different samples: (a) 237 smokers paid to participate in laboratory experiments in Ann Arbor, Michigan; data were collected via telephone screen and approximately 2 weeks later via questions embedded in a written history; and (b) 36 smokers hospitalized for depression in Paris; data were collecled via a written questionnaire upon admission and again after 3 weeks of treatment for depression. Subjects in both samples were regular smokers who were not attempting to quit and who were not in treatment for smoking cessation. Reliability data are also presented for the FTND and compared with FTQ data collected in a subsample of 60 subjects in the American database who received both versions of the questionnaire. Analyses of internal consistency, using Cronbach's alpha, and external validity, using years smoked (associated with increases in intake; Russell, 1979), cotinine (a nicotine metabolite that serves as a biological index of intake), and scores on the CSM "addictive" factor (Russell et al., 1974) as criterion variables, are reported briefly.

$M$ E T H O D

\section{Subjects}

Subjects were drawn from two separate samples: (a) 237 smokers $(43.5 \%$ female: age $[M \pm S D], 28.9 \pm 6.6$ years), in good health (including no history of psychopathology) and not on psychoactive medications, who were paid to participate in experiments in our laboratory between 1986 and the present. A subsample of 60 smokers $(25.0 \%$ female; age $28.7 \pm 6.6$ years) received the FTND as well. Although subject selection criteria varied across experiments, most were recruited for being moderate smokers who smoked at least 15 cigarettes per day and who had smoked for at least 3 years, and for being in the 20 to 45 age range. (b) 36 smokers $(66.7 \%$ female; age $36.3 \pm 9.6$ years) meeting the DSM-III-R criteria of major depression, hospitalized in the Salpêtrière Hospital (Paris).

\section{Procedure}

For the American laboratory sample, test-retest data were collected initially via telephone screen and subsequently $(15.0 \pm 31.3$ days later) via questions embedded in a written history. More recently, sufficient data have been collected to permit calculation of scores for both the FTQ and the FTND. Also available for many of 
these subjects were baseline cotinine levels assayed using HPLC (Hariharan, VanNoord, \& Greden, 1988), number of years smoked, and scores on the "addiclive" scale of a brief version of the CSM (Russell et al., 1974) that includes only the three items that loaded highest on each factor and that retains the factor structure of the original (Tate, Pomerleau, \& Pomerleau, in press). Because depression has been associated with smoking status (Glassman et al., 1990), we also included in our analyses scores on the Center for Epidemiological Studies Depression Scalc (CES-D; Weissman, Sholomakis, Pottenger, Prushoff, \& Locke, 1970). (Data were drawn from a database including baseline and demographic variables collected at screening or via take-home packets. Because these packets varied somewhat depending on the study and the time at which they were collected, data for some of the variables included as validators were not available for all subjects.)

For the French sample, the FTQ was filled out as part of a battery of questionnaires administered in the context of a study of smoking behavior in depressed subjects. Each subject was assessed upon admission, before administration of antidepressant treatment and in some instances after a placebo wash-out period. An extensive psychiatric interview, including the Hamilton Rating Scale for Depression (HRSD; Hamilton, 1967), was conducted by an experienced clinician in order to evaluate intensity of symptomatology, following which patients were asked to complete the questionnaires. Subjects were reassessed just prior to discharge, when they were judged as normothymic by the clinicians, after at least 3 weeks' antidepressant treatment; for the majority, assessment was made on Day 21. The procedure was identical to that at Day 0. The CSM was also administered; for comparability across the two samples, only the three items administered to the American sample were used.

Validity data are presented only for the telephone screen data in the American sample and for the admission interview for the French sample. Results in both instances were similar for the second administration.

R E S U L T S

\section{$F T Q$}

Test-retest correlations for the two samples, for both the full questionnaire and the individual items, are shown in Table 1 . No decrease in test-retest reliability was observed as a function of time elapsed between the two administrations in the American sample, as determined by correlating the absolute difference between the two scores with the amount of time between administration ( $n=196 ; r=-.10$, NS).

In the American sample, there was a small but statistically significant decrease $(t[236]=5.23, p<.001)$ in FTQ scores between Time 1 (telephone screen; $7.3 \pm 1.8$ ) and Time 2 (written history; $6.9 \pm 2.0$ ). Individual items that contributed significantly to this drop were item 1 (time to first cigarette), item 2 (forbidden cigarettes), item 3 (which cigarette would you most hate to give up?), and item 6 (smoke if ill). In the French sample, scores on the first administration (5.75 \pm 2.3$)$ did not differ significantly from scores on the second $(6 \pm 2.5)$, nor did any item score differ significantly between administrations.

Cronbach's alpha for our laboratory subjects has been previously reported (Pomerleau et al., 1990); with the additional subjects in the current database, Cronbach's alpha for the FTQ was .47. For the French sample, Cronbach's alpha was .61.

A significant correlation between cotinine and FTQ score in our laboratory sub- 
Table 1. Test-retest correlations for total FTQ score and individual items in two samples

\begin{tabular}{lcc}
\hline & $\begin{array}{c}\text { American sample } \\
(n=237)\end{array}$ & $\begin{array}{c}\text { French sample } \\
(n=36)\end{array}$ \\
\hline FTQ (total) & $+.783^{* *}$ & $+.845^{* *}$ \\
FTQ1 (time to 1st cigaretle) & $+.717^{* *}$ & $+.875^{* *}$ \\
FTQ2 (forbidden cigarettes) & $+.514^{* *}$ & $1.883^{* *}$ \\
FTQ3 (most hate to give up) & $+.663^{* *}$ & $+.837^{* *}$ \\
FTQ4 (cigarettes/day) & $+.749^{* *}$ & $+.659^{* *}$ \\
FTQ5 (morning smoking) & $+.613^{* *}$ & $+.516^{*}$ \\
FTQ6 (smoke if ill) & $+.577^{* *}$ & $+.887^{* *}$ \\
FTQ7 (nicotine yield) & $+.802^{* *}$ & $+.797^{* *}$ \\
FTQ8 (inhale?) & $+.587^{* *}$ & $+.930^{* *}$ \\
\hline
\end{tabular}

$p<.005: *^{*} p<.001$

jects has been previously reported (Pomerleau et al., 1990). This correlation has been sustained with the addition of more subjects to our database $(n=136 ; r=+.35, p<$ $.001)$. Correlation with number of years smoked $(n=214)$ was $+.38(p<.001)$. Scores on the CSM "addictive" factor were significantly correlated with FTQ in both the American sample $(n=216 ; r=+.40, p<.001)$ and in the French sample $(n=35 ; r=+.62, p<.001)$.

In the American sample $(n=183)$, no correlation was detected between CES-D and FTQ scores $(r=+.02$, NS). Likewise, in the French sample, no association was detected hetween FTQ score and severity of depression as measured by the HRSD $(r=-.03, \mathrm{NS})$.

\section{FTND}

Test-retest correlations for both the FTQ and the FTND, in the subsample of 60 who took both questionnaires, are shown in Table 2 . There was no significant difference between the first and second administration for either the FTQ $(M \pm S D$ for first administration, $6.9 \pm 2.2$; for second administration, $6.7 \pm 2.1 ; t[59]=.83$, NS) or the FTND (first administration, $5.3 \pm 2.4$; second administration, $5.1 \pm 2.4 ; t[59]=$ $1.44, \mathrm{NS})$.

Cronbach's alpha was .58 for the FTQ and .64 for the FTND. Correlation with cotinine $(n=27)$ was $+.45(p<.05)$ for the FTQ and $+.39(p<.05)$ for the FTND. Correlation with number of years smoked was $+.57(p<.001)$ for the FTQ and +.52 $(p<.001)$ for the FTND. Correlation with the CSM "addictive" factor $(n=55)$ was $+.56(p<.001)$ for the FTQ and $+.53(p<.001)$ for the FTND. No relationship with depression $(n=59)$ was detected for either the FTQ $(r=-.18, \mathrm{NS})$ or the FTND $(r=-.24, \mathrm{NS})$.

\section{I S C U S I O N}

In both the American and French samples, the FTQ showed very satisfactory test-retest reliability. A high correlation emerged in the American sample despite the use of alternate forms (telephone vs. paper-and-pencil), which might be expected to attenuate the association. Individual items were also well-correlated. 
Table 2. Test-retest correlations for FTQ and FTND scores (total and items in the same sample)

\begin{tabular}{lcc}
\hline & $\begin{array}{c}\text { FTQ } \\
(n=60)\end{array}$ & $\begin{array}{c}\text { FTND } \\
(n=60)\end{array}$ \\
\hline Total & $+.828^{* *}$ & $+.882^{* *}$ \\
Time to 1st cigarette & $+.926^{* *}$ & $+.817^{* *}$ \\
Forbidden cigarettes $^{\mathrm{a}}$ & $+.493^{* *}$ & $+.493^{* *}$ \\
Most hate to give up $^{\mathrm{a}}$ & $+.665^{* *}$ & $+.665^{* *}$ \\
Cigarettes/day $_{\text {Morning smoking }}^{\mathrm{a}}$ & $+.892^{* *}$ & $+.970^{* *}$ \\
Smoke if ill & $+.506^{* *}$ & $+.506^{* *}$ \\
Nicotine yield & $+.629^{* *}$ & $+.629^{* *}$ \\
Inhale? & $+.727^{* *}$ & - \\
\hline
\end{tabular}

aThese items are used and scored identically in both questionnaires.

${ }^{* *} p<.001$.

The FTQ performed equally well in two samples that differed with respect to presence of depression and sociocultural factors. Of particular note is the difference in smoking rate, which is currently around $25 \%$ in the United States (National Health Interview Survey, 1991) and $40 \%$ in France (Molimard et al., 1992). In fact, smoking prevalence in France today is virtually identical to that in the United States in 1965 . only a year after the Report of the Surgeon General's Advisory Committee on Smoking and Health (USPHS, 1964) publicizing the health hazards of smoking. In a society in which smoking is not seriously discouraged, one would expect considerably more "discretionary" smoking than in a society characterized by extensive public health campaigns and social sanctions. Our findings thus suggest that the FTQ is potentially useful in a broad spectrum of populations.

A small but consistent decrease in score between the first and second administration occurred in the American, but not in the French, sample. A possible explanation is that, because our subjects were recruited for being moderately dependent, the change simply represents regression to the mean. It is also possible, however, that the use of alternate forms influenced test results.

Although the nature of the relationship between depression and degree of dependence remains to be delineated, our results suggest that treatment of depression does not affect the reliability of the FTQ.

Test-retest reliability of the FTND was as good as or better than that of the FTQ. We likewise replicated Heatherton et al.'s (1991) results showing a somewhat higher Cronbach's alpha in the FTND than in the FTQ when calculated in the same group of subjects. Our rough measures of external validity, however, did not demonstrate any clearcut superiority for the FTND in this relatively small sample.

It should be noted that one of the problems relating to validation of either the FTQ or the FTND is the lack of either "hard" (biological) measures of dependence or a universally agreed-upon set of criteria that would allow us, say, to comment on the sensitivity and specificity of the test, or to establish cutoffs for what Shiffman (e.g., 1990) has referred to as nondependent smokers. In fact, we may be demanding more of the test, in terms of internal consistency and construct validity, than we demand of the concept of "physical dependence" itself — because there is no clearcut evidence that chronic tolerance (both pharmacokinetic and pharmacodynamic), acute 
tolerance, withdrawal symptomatology, drug self-administration (as measured by intake), ete., are unidimensional. The price we pay for achieving a "homogeneous set" of questions (Heatherton et al., 1991), for example, may be a loss of information about actual drug intake; and though no one would argue that intake and dependence are coterminous, it would be equally rash to say that strength of drug-seeking behavior is irrelevant to dependence. An extensive consideration of the nature of dependence is outside the scope of this article; our point is that major improvements in the test will probably follow rather than precede refinements in our understanding of dependence itself. Meanwhile, we may sometimes find ourselves in the position of defining nicotine dependence (to paraphrase an only partly facetious definition of another elusive concept, intelligence) as "that which the FTND measures."

\section{R E F E R E N C E S}

Fagerstrom, K. O. (1978). Measuring degree of physical dependence to tobacco smoking with reference to individualization of treatment. Addictive Behaviors, 3, 235-241.

Fagerstrom, K. O. (1982). Effects of a nicotine-enriched cigarette on nicotine titration, daily cigarette consumption, and levels of carbon monoxide, cotinine, and nicotine Psychophamacology, 77. 164167.

Fagerstrom, K. O., \& Schneider, N. (1989). Measuring nicotine dependence: A review of the Fagerstrom Tolerance Questionnaire. Journal of Behavioral Medicine, 12, 159-182.

Glassman, A. H., Helzer, J. E., Covey, L. S., Cottler, L. B.. Stetner, F.. Tipp. J. E., \& Johnson, J. (1990). Smoking, smoking cessation, and major depression. JAMA, 264, 637-640.

Hamilton, M. (1967). Development of a rating scale for primary depressive illness. British Journal of Social and Clinical Psychology, 6, 278-296.

Hariharan, M., VanNoord, T., \& Greden, J. F. (1988). A high-performance liquid-chromatographic method for routine simultaneous determination of nicotine and cotinine in plasma. Clinical Chemistry, 34, 724-729.

Heatherton, T. F. Kozlowski, L. T., Frecker, R. C., \& Fagerstrom, K. O. (1991). The Fagerstrom test for nicotine dependence: A revision of the Fagerstrom tolerance questionnaire. British Jom of Addiction, 86, 1119-1127.

Ikard. F. F., \& Tomkins, S. (1973). The experience of affect as a determinator of smoking behavior: A series of validity studies. Journal of Abnormal Psychology, 81, 172-181.

Lichtenstein, E., \& Mermelstein, R. J. (1986). Some methodological cautions in the use of the tolerance questionnaire. Adtlictive Behavions, 11,439-442.

Lombardo, T. W., Hughes, J. R., \& Fross, J. D. (1988). Failure to support the validity of the Fagerstrom Tolerance Questionnaire as a measure of physiological tolerance to nicotine. Addictive Behaviors, 13 , $87-90$.

Molimard. R.. Le Guilloux. F., Poullaouec. D., \& Guedon. C. (1992). Dépendances chezz les malades hospitalisés. Semaine des Hopitaux, 68. 1314-1316.

Moore. B. L., Schneider. M. A., \& Ryan. J. J. (1987). Fagerstroms's Tolerance Questionnaire: Clarification of item and scoring ambiguities. Addictice Bathaters. 12, 67-68.

National Health Interview Survey. (1991). Data compiled by the Office on Smoking and Health. Center for Disease Control.

Pinto. R. P.. Abrams. D. B. Monti. P. M. . \& Jacobus. S. 1. (19871. Nicotine dependence and likelihood of quitting smoking. Addictive Behavions, 12.371-374.

Pomerleau, C. S., Majchrzak, M. J., \& Pomerleau. O. F. (1989). Nicotine dependence and the Fagerstrom Tolerance Questionnaire: A brief review. Journal of Substance Abuse, 1, 471-477.

Ponerleau, C. S., Pomerleau, O. F., Majch Lak. M. J., Kloska, D. D., \& Malakuti, R. (1990). Relationship between Nicotine Tolerance Questionnaire scores and plasma cotinine. Addicfive Behaviors. 15. $73-80$.

Pomerleau. O. F., Fertig. J.. \& Shanahan. S. O. (1983). Nicotine dependence in cigarette smoking: An empirically based. multivariate model. Pharmacology Biochemistry \& Behavior, 19, $291-299$.

Russell, M. A. H. (1979). Tobacco dependence: Is nicotine rewarding or aversive'? NIDA Research Monograph \#23. Washington, DC: U.S. Government Printing Office.

Russell, M. A. H.. Peto, J., \& Patel. U. A. (1974). The classification of smoking by factorial structure of motives. Jonmal of the Royal Statistical Society, 137, 313-346.

Shiffman, S., Fischer. L. B., Zettler-Segal, M., \& Benowitz, N. L. (1990). Nicotine exposure among nondependent smokers. Archives of General Prychiar., 47, 3.33-336.

Swan, G. F.. Ward. M. M., \& Jack, L. (1991). The Fagerstrom Tolerance Questionnaire: A prychometric analysis. Presented at the Annual Meeting of the Society of Behavioral Medicine. Washington. DC. 
Tate, J. C., Pomerleau, C. S., \& Pomerleau, O. F. (in press). Pharmacological and non-pharmacological smoking motives: A replication and extension. Addiction.

Tomkins, S. (1966). Psychological model for smoking behavior. American Journal of Public Health. 56. $217-220$.

U.S. Public Health Service (1964). Smoking and health. Report of the Advisory Committee to the Surgeon General of the Public Health Service. Washington, DC: PHS Publication No. 1103. U.S. Department of Health, Education, and Welfare, Public Health Service, Center for Disease Control.

Weissmann, M. M., Sholomakis, D., Pottenger, M., Prushoff, B. A., \& Locke. B. Z. (1977). Assessing depressive symptoms in five psychiatric populations: A validation study. American Jommal of Epidemiology, 106, 203-214. 